\title{
Embolia de shunt carotídeo temporário para a artéria poplítea
}

\author{
Temporary carotid shunt embolism for popliteal artery
}

Leonardo Pires de Sá Nóbrega', Alcides José Araújo Ribeiro', Cláudio Eluan Kalume²

\begin{abstract}
Resumo
O deslocamento de um shunt para um ramo arterial mais distal, percorrendo um longo trajeto, é uma complicação rara. A ultrassonografia vascular pode se apresentar como uma excelente modalidade diagnóstica para identificar o sítio de embolização de corpos estranhos intravasculares. Os autores relatam um caso raro de migração de um shunt temporário, implantado na carótida comum esquerda, para artéria poplítea esquerda. Descrevem, ainda, a utilização do ecocolor doppler arterial que, além de localizar o ponto de embolização, com uma dermatografia exata, foi fundamental para que a abordagem cirúrgica transcorresse com facilidade através de uma via de acesso restrita e precisa.
\end{abstract}

Palavras-chave: artéria carótida primitiva; artéria poplítea; ultrassonografia doppler em cores.

\begin{abstract}
The displacement of a shunt to a more distal arterial branch, along a path, is a rare complication. Vascular ultrasound can be presented as an excellent diagnostic modality for identifying the site of embolization of intravascular foreign bodies. The authors report a rare case of temporary shunt migration, implanted in the left common carotid artery, to left popliteal artery. The authors also describe the use of arterial vascular ultrasound to find the point of embolization, with an exact dermatography, that was essential to an easily surgical approach through a restrict and precise access way.
\end{abstract}

Keywords: carotid common artery; popliteal artery; ultrasonography doppler color.

\section{Introdução}

A identificação de corpos estranhos (CE) intravasculares é rara. A grande maioria dos casos de embolização tem como ponto de origem o sistema venoso profundo e a migração se dá, em geral, para estruturas como a veia cava superior, ventrículo direito, artéria pulmonar ou combinação dessas estruturas ${ }^{1}$. Menos frequentemente, os CE são encontrados na aorta ou em algum ramo arterial, entretanto, a maior freqüência de procedimentos endovasculares percutâneos proporcionou um aumento de casos de CE intra-arteriais, sendo o mais comum a embolização de stents, cateteres e fragmentos de fio guia $^{2}$. O deslocamento de um shunt temporário para um ramo arterial mais distal é uma complicação rara ${ }^{3}$. Este relato de caso ilustra a experiência dos autores com a utilização da ultrassonografia vascular para localizar um fragmento de sonda nasogástrica, utilizada como derivação carotídea temporária, que migrou para artéria poplítea esquerda.

\section{Relato de caso}

Paciente masculino, 36 anos, vítima de múltiplas perfurações por arma branca após tentativa de autoextermínio, atendido no pronto-socorro pela equipe da cirurgia geral de um hospital de atendimento secundário. Foram diagnosticadas lesões perfurocortantes em abdome, tórax e região cervical esquerda; foi realizado o atendimento inicial com drenagem torácica, além de laparotomia exploradora e cervicotomia esquerda.

Durante a cervicotomia foram identificadas lesões de laringe e da artéria carótida comum esquerda (ACCE). O cirurgião geral, após dissecção dos cotos proximal e distal da ACCE, introduziu um shunt carotídeo temporário confeccionado com sonda nasogástrica $\mathrm{n}^{\circ} 16$, medindo $8,5 \mathrm{~cm}$

Trabalho realizado no Hospital de Base do Distrito Federal (HBDF) - Brasília (DF), Brasil.

${ }^{1}$ Médico-assistente da Unidade de Angiologia e Cirurgia Vascular, HBDF e da Clínica de Veias - Brasília (DF), Brasil.

${ }^{2}$ Médico-chefe da Unidade de Angiologia e Cirurgia Vascular, HBDF - Brasília (DF), Brasil.

Conflito de interesse: nada a declarar.

Submetido em: 27.03.11. Aceito em: 15.09 .11

J Vasc Bras. 2011;10(4):330-334. 
de comprimento, fixada com fios de seda no coto proximal e distal. Dessa forma, foi mantida satisfatoriamente a perviedade do vaso. A lesão da laringe foi tratada com rafia simples e traqueostomia.

Após a estabilização hemodinâmica, o paciente foi encaminhado à unidade de angiologia e cirurgia vascular do hospital terciário da rede para tratamento especializado da lesão da ACCE. Durante a cirurgia, observou-se que não haveria necessidade de interposição de enxerto, uma vez que seria possível um reparo primário através da confecção de uma anastomose término-terminal dos cotos da ACCE. Entretanto, no momento da liberação da fixação do shunt carotídeo temporário, ocorreu a migração inadvertida do mesmo para o interior do arco aórtico. Após o término da cirurgia proposta, o paciente encontrava-se estável e com todos os pulsos palpáveis em membros superiores e inferiores.

Para identificação do sítio de embolização do shunt temporário foi proposta uma varredura abdominal, pélvica e de membros inferiores com ecocolor doppler arterial (ECDA). Este exame realizado com um equipamento Aplio (Toshiba America Medical Systems, Inc. Tustin, Califórnia, EUA), utilizando-se transdutores lineares multifrequenciais de 6-11 e 6,2-12,0 MHz. O CE foi identificado no interior da artéria femoral superficial esquerda (AFSE). Neste estudo, foi feita uma avaliação inicial com o modo-B (Figura 1), sendo possível não só constatar a presença do CE no interior da AFSE, mas também a sombra acústica que o acompanhava em todo o seu trajeto. Em seguida, associando-se o power doppler (Figura 2), foi possível identificar um estreitamento do fluxo sanguíneo ao passar pelo interior do CE, confirmando a sua perviedade.

Como a intervenção cirúrgica para retirada do $\mathrm{CE}$ só foi possível após algumas horas, foi indicado um novo exame de ecografia vascular com o objetivo de demarcar a sua localização exata e facilitar o ato operatório. O segundo exame constatou que o CE já havia migrado para a artéria poplítea proximal esquerda e em sua realização foi utilizado um transdutor convexo multifrequencial de 1,9-6,0 $\mathrm{MHz}$ (Figuras 3 e 4).

Assim, com a dermatografia atualizada precisamente (Figura 5), os cirurgiões realizaram uma incisão relativamente pequena e não encontraram qualquer dificuldade em acessar, por via medial, o terço proximal da artéria poplítea esquerda e retirar o CE do seu lúmen, através de arteriotomia transversa (Figuras 6, 7 e 8). Foram introduzidos cateteres de Fogarty ${ }^{\oplus} \mathrm{n}^{\circ} 3$ e 4 (Edwards Lifesciences Macchi, São Paulo, SP, Brasil), sendo observada a saída de trombos de aspecto recente, seguida de instilação proximal e distal de

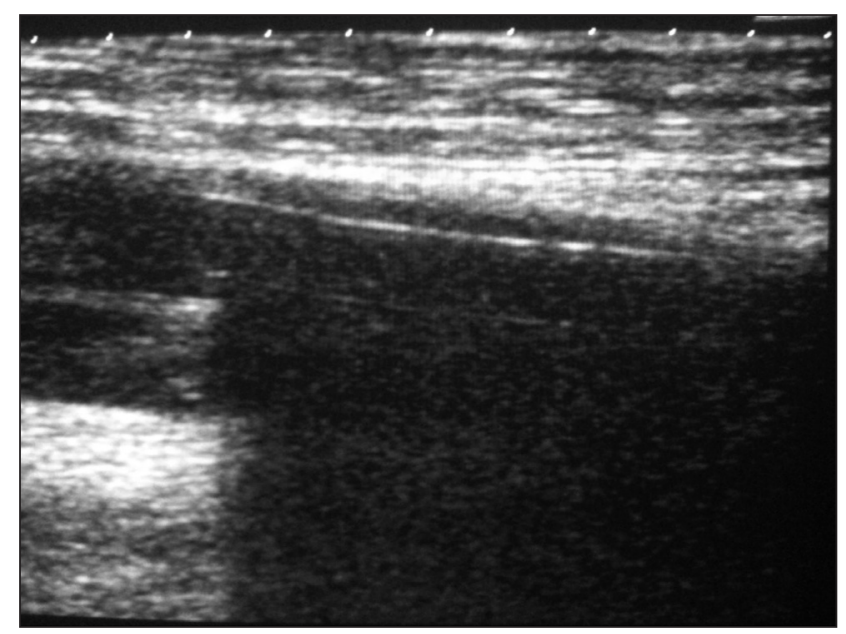

Figura 1. Modo B: corpo estranho no interior da artéria femoral superficial esquerda (observar sombra acústica posterior).

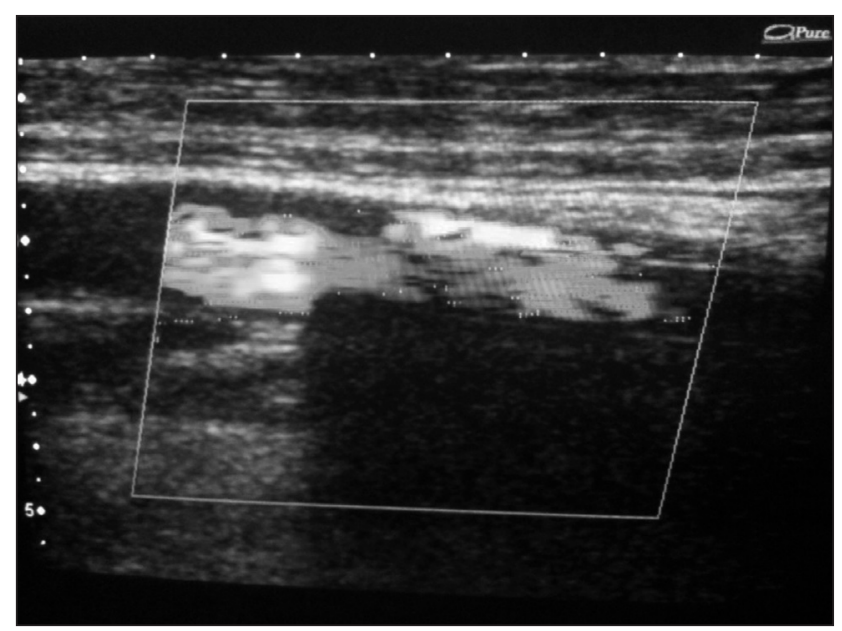

Figura 2. Modo B + power doppler: corpo estranho no interior da artéria femoral superficial esquerda (observar estreitamento do fluxo ao passar pelo interior do $\mathrm{CE}$ ).

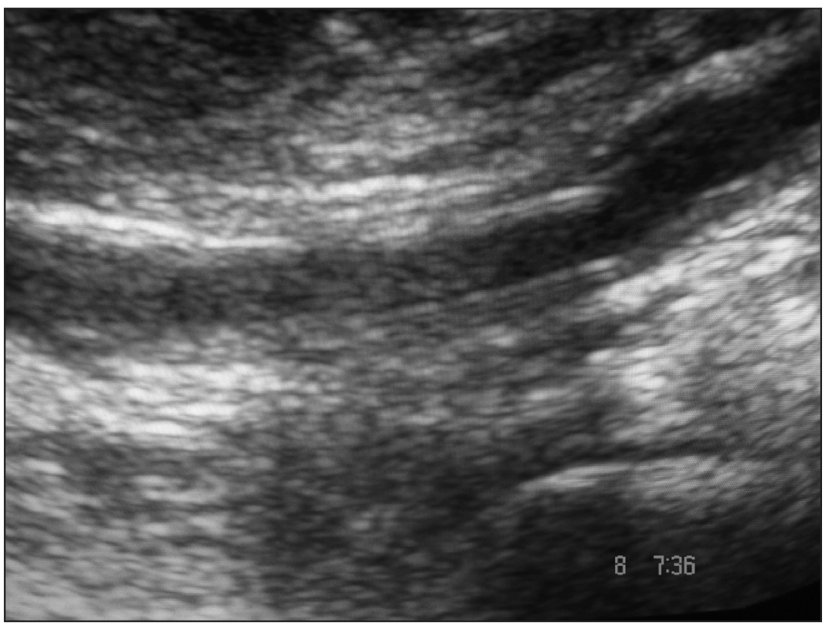

Figura 3. Modo B: extremidade distal do corpo estranho no interior da artéria poplítea esquerda. 


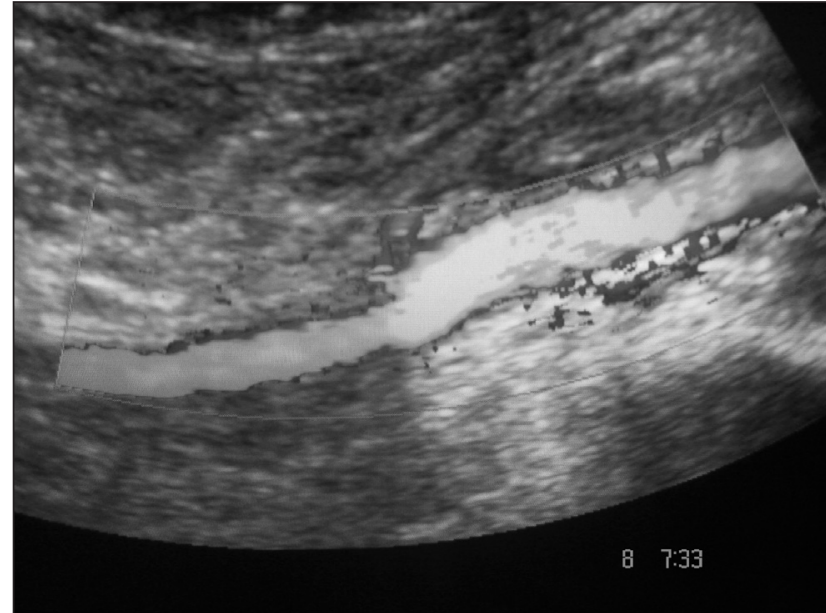

Figura 4. Modo B + power doppler: extremidade distal do corpo estranho no interior da artéria poplítea esquerda.

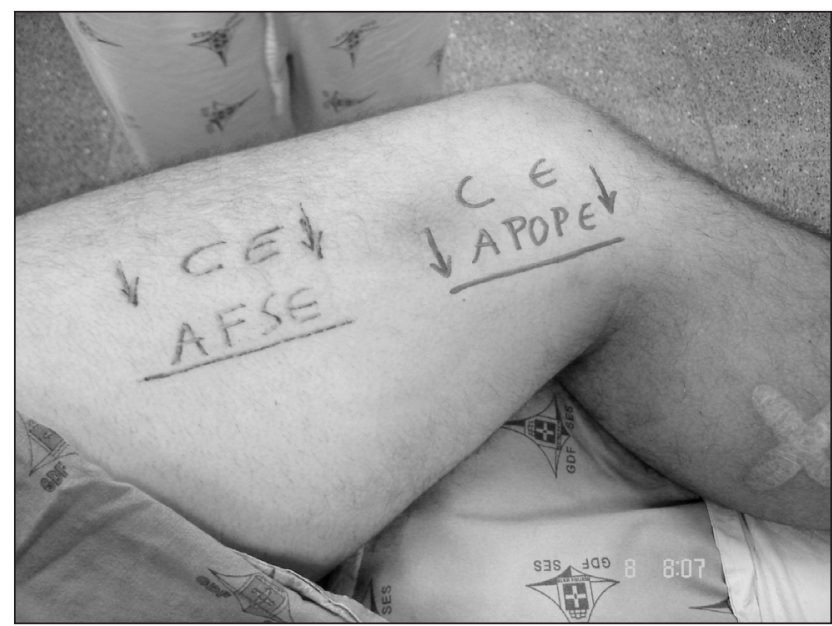

Figura 5. Dermatografia demonstrando a migração do corpo estranho da artéria femoral superficial esquerda para a artéria poplítea ipsilateral.

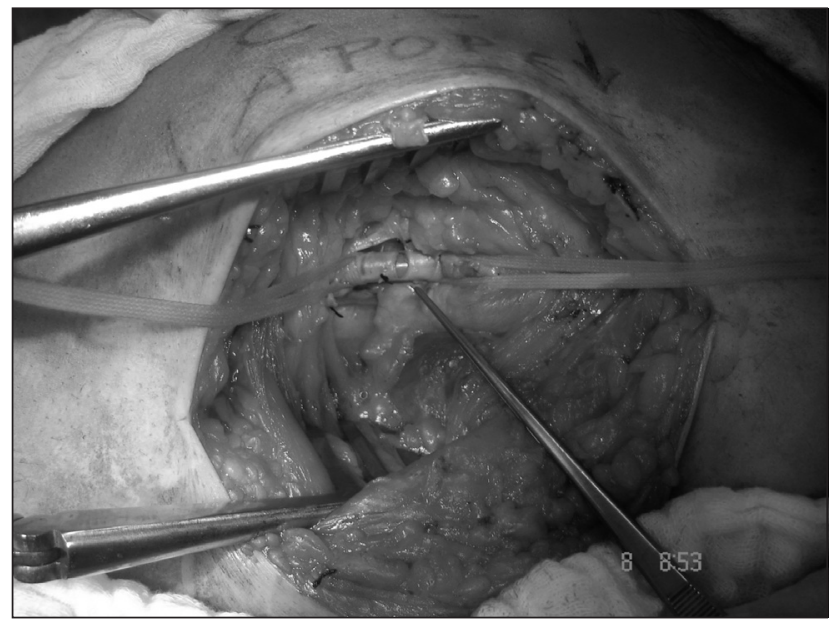

Figura 6. Arteriotomia transversa da poplítea esquerda com a sonda nasogástrica no seu lúmen (observar que a incisão foi compatível com a marcação dermatográfica previamente realizada).

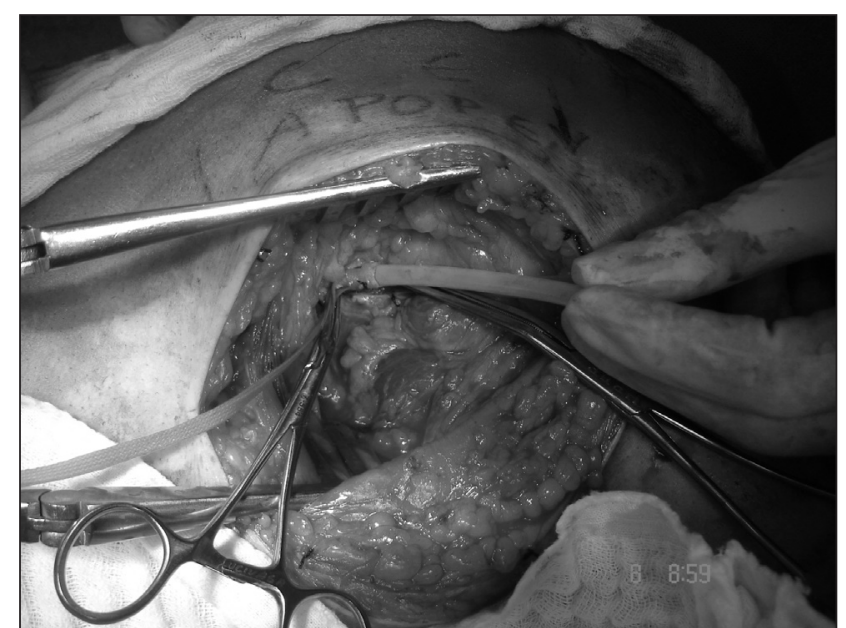

Figura 7. Extração do corpo estranho do interior do lúmen da artéria poplítea esquerda.

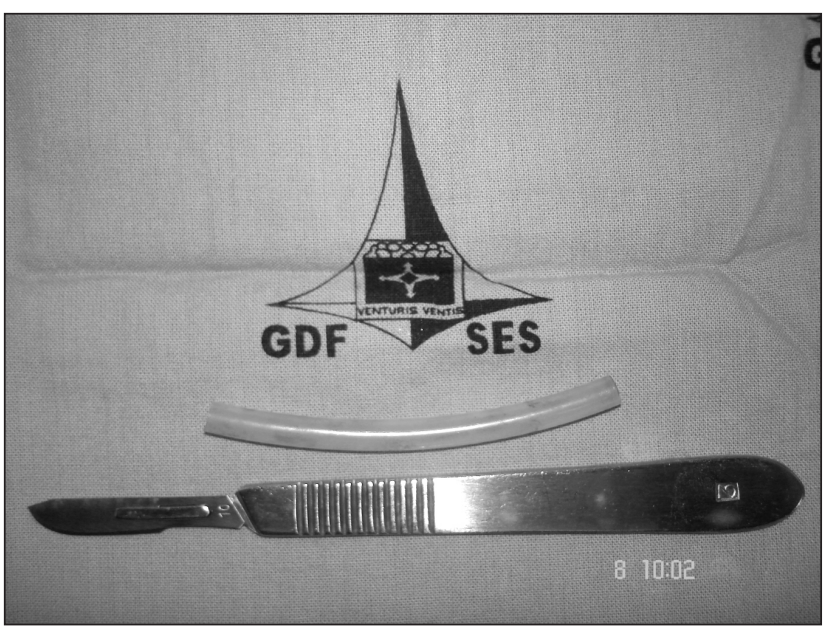

Figura 8. Corpo estranho: segmento de sonda nasogástrica.

solução salina de heparina. Ao término do procedimento, o paciente permanecia com os pulsos distais palpáveis no membro inferior esquerdo e, após $24 \mathrm{~h}$, retornou aos cuidados da cirurgia geral para dar continuidade ao seu tratamento, em uso de anticoagulação pós-operatória e sem seqüelas neurológicas.

\section{Discussão}

Os shunts intravasculares são condutos artificiais colocados dentro do lúmen vascular de um segmento lesado, para restabelecer temporariamente a irrigação sanguínea, até que seja possível realizar a reconstrução definitiva, tratando-se de uma técnica apropriada de controle de dano. A equipe da cirurgia geral utilizou um segmento de sonda nasogástrica no 16 , com cerca de $8,5 \mathrm{~cm}$ de comprimento. A escolha do material do shunt é uma questão de preferência individual, tendo em 
vista que qualquer tubo sintético estéril, liso, rígido e com calibre apropriado, pode servir como conduto temporário ${ }^{3}$.

As principais complicações dos shunts intravasculares temporários são trombose e deslocamento, essa primeira complicação é a mais freqüente e pode representar a evolução natural da técnica, caso o shunt não seja substituído pela revascularização definitiva. Já a migração do shunt para um ramo arterial mais distal é uma complicação rara e com poucos casos descritos na literatura médica ${ }^{3}$.

A embolização no sistema vascular depende de vários fatores, como: rota de entrada, gravidade e posição do paciente no momento do acidente. O ponto final do CE está relacionado com o tamanho e rigidez do material, bem como com os padrões de fluxo do vaso (Figura 9). Migrações de CE de longa distância são raras na literatura médica ${ }^{1}$.

Normalmente o quadro clínico da embolização arterial depende da localização do CE e as manifestações agudas são bem conhecidas pelos médicos especialistas: dor, parestesia, frialdade, palidez da extremidade e ausência de pulsos distalmente ao ponto de oclusão, que não foram identificadas no paciente, uma vez que o CE apresentava um lúmen pérvio proporcionando-o uma condição assintomática ${ }^{4}$.

Como não havia nenhum indicativo clínico da topografia do $\mathrm{CE}$, foi recomendada uma busca com o ECDA, que já foi utilizado em algumas situações para a localização de $\mathrm{CE}$ intra-arteriais, principalmente para pesquisa de stents que migraram de seus sítios originais ${ }^{5-7}$. Na literatura médica especializada utilizada pelos autores, não foi encontrado nenhum relato de caso onde esse método diagnóstico tenha sido usado em uma varredura abdominal, pélvica e nos membros inferiores para a localização de um shunt temporário que tenha embolizado para uma artéria mais distal. A arteriografia seria uma boa alternativa caso o CE não fosse localizado através do ECDA, pois apesar do CE ser radiotransparente, através de sinais indiretos, o mesmo poderia ser identificado, porém com maior custo e morbidade associada ao procedimento ${ }^{1,2}$.

Os CE da aorta torácica ou abdominal geralmente migram para artérias dos membros inferiores. A perna esquerda é três vezes mais acometida que a direita e isso talvez seja resultado de uma angulação menos aguda entre artéria ilíaca comum esquerda e o eixo central da aorta $\left(30^{\circ}\right)$, em relação ao lado direito $\left(45^{\circ}\right)^{8,9}$.

Como o CE migrou inicialmente para a AFSE, a sua localização foi facilmente determinada através do ECDA de membros inferiores, com o uso de transdutores lineares. A identificação de fluxo no interior do CE, com a aplicação do power doppler, confirmou a perviedade da AFSE, justificando a presença de pulsos amplos em artérias pediosa e tibial posterior esquerdas.

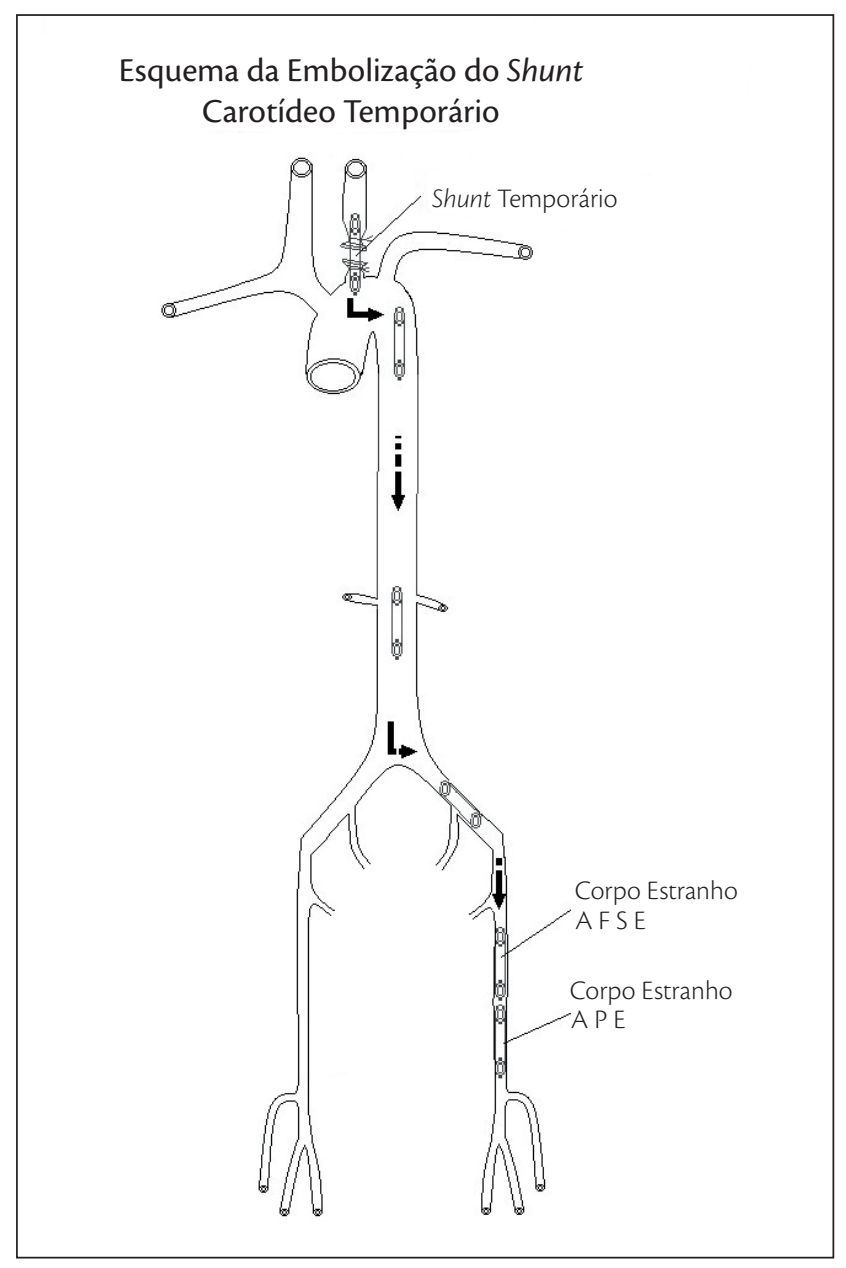

Figura 9. Esquema do longo trajeto percorrido pelo shunt temporário.

Como não houve possibilidade de intervenção cirúrgica imediata, a realização de um novo exame de ecografia vascular foi extremamente oportuna, uma vez que constatou a embolização do corpo estranho para a artéria poplítea. No segundo exame, houve uma dificuldade técnica durante a identificação da artéria poplítea proximal, devido à musculatura exuberante da coxa, que foi contornada com a utilização de um transdutor convexo multifrequencial de 1,9-6,0 MHz. A confecção de uma dermatografia precisa foi fundamental para que a abordagem cirúrgica transcorresse com facilidade, não sendo necessária a ampliação da via de acesso. Já a retirada do CE por via percutânea não seria possível devido à ausência de materiais capazes de mobilizá-lo e também devido ao seu tamanho e calibre ${ }^{2,3}$.

Concluímos que o shunt carotídeo temporário é uma boa forma para manter a perviedade do sistema carotídeo e a ecografia vascular com doppler é uma modalidade diagnóstica não invasiva, rápida, de menor custo e capaz de identificar com facilidade a topografia dos locais de 
embolização de CE, além de proporcionar uma dermatografia exata que permite a realização da intervenção cirúrgica através de incisões menores e mais precisas.

\section{Referências}

1. Uflacker R, Lima S, Melichar AC. Intravascular foreign bodies: percutaneous retrieval. Radiology. 1986;160(3):731-5.

2. Małecka B, Kutarski A, Zabek A, et al. Percutaneous removal of endocardial implantable cardioverter-defibrillator lead displaced to the right pulmonary artery. Cardiol J. 2010;17(3):293-8.

3. Hirshberg A, Scott BG. Controle de danos no trauma vascular. In: Rich NM, Mattox KL, Hirshberg A, editores. Trauma Vascular. Rio de Janeiro: DiLivros. $2^{\mathrm{a}}$ ed. 2006. p. 184-5.

4. Iyisoy A, Kursaklioglu H, Celik T, et al. Coronary stent embolization into right common femoral artery - the role of computed tomography angiography. Clin Cardiol. 2009 Jul;32(7):E9.

5. Ashar RM, Huettl EA, Halligan R. Percutaneous retrieval of a Wallstent from the pulmonary artery following stent migration from the iliac vein. J Interv Cardiol. 2002;15(2):101-6.

6. Wijesinghe LD, Coughlin PA, Gill K. An unusual cause of femoral embolus. Cardiovasc Surg. 2000;8(4):287-8.
7. Prabhudesai A, Khan MZ. An unusual cause of femoral embolism: angioseal. Ann R Coll Surg Engl. 2000;82(5):355-6.

8. Patel KR, Cortes LE, Semel L, Sharma PV, Clauss RH. Bullet embolism. J Cardiovasc Surg (Torino). 1989;30(4):584-90.

9. Burkitt DS, Dhasmana JP, Mortensen NJ, Wisheart JD. 'Bullet embolism' to the popliteal artery following air rifle injury of the thoracic aorta. Br J Surg. 1984;71(1):61.

Correspondência
LEPS 715/915 - Conjunto A - Bloco D - Ed. Pacini, salas 317 e 318 - Asa Sul
CEP: 70.390-155 - Braślia (DF), Brasil
E-mail: leonardo.nobrega@gmail.com
Contribuições dos autores
Concepção e desenho do estudo: LPSN
Análise e interpretação dos dados: LPSN
Coleta de dados: LPSN
Redação do artigo: LPSN
Revisão crítica do texto: AJAR
Aprovação final do artigo*: LPSN, AJAR, CEK
Análise estatística: não se aplica
Responsabilidade geral pelo estudo: LPSN
Informações sobre financiamento: LPSN

